\title{
PRESENTACIÓN
}

\section{La nueva ola de integración en Asia-Pacífico}

Melba E. Falck Reyes ${ }^{1}$

Opinión invitada

José Jaime López Jiménez ${ }^{2}$

Santiago Aceves Villalvazo ${ }^{3}$

DOI: $10.32870 /$ mycp.v2i4.401

Editores

Tres acuerdos regionales de integración económica se están negociando actualmente en Asia-Pacífico: el Acuerdo de Asociación Transpacífico (TPP, por sus siglas en inglés), el Acuerdo Regional de Asociación Económica Comprehensivo (RECEP, por sus siglas en inglés) y la Alianza del Pacífico (AP). En los tres hay varios países involucrados que cuentan con acuerdos bilaterales entre sí, y que ahora buscan profundizar esa integración con nuevas reglas. No obstante, el modelo empleado por cada uno difiere en intensidad y forma.

El TPP desde su inicio tuvo un carácter transpacífico al involucrar, por un lado a Chile y, por otro, a Nueva Zelanda y Singapur. A partir de entonces se han ido incorporando nuevos miembros hasta completar las 12 economías que lo conforman actualmente. Teóricamente este grupo, bajo el liderazgo de

1. Profesora-investigadora del Departamento de Estudios del Pacífıco, del Centro Universitario de Ciencias Sociales y Humanidades de la Universidad de Guadalajara, y miembro del SNI. ORCID http://orcid.org/0000-0003-4926-0594

2. Profesor-investigador del Departamento de Estudios del Pacífico, del Centro Universitario de Ciencias Sociales y Humanidades de la Universidad de Guadalajara.

3. Profesor del Instituto Tecnológico y de Estudios Superiores de Occidente (ITESO). 
Estados Unidos y después de 17 rondas de negociaciones, busca concluir un acuerdo de integración que esté a la altura de los desafíos del siglo XXI. Por ende, el TPP no sólo se propone ser un acuerdo comprehensivo en cuanto a los sectores que abarcará (ningún sector debe quedar fuera), sino en relación con los "nuevos" temas incluidos. Así, en los 29 capítulos en negociación, además de los temas tradicionales de acceso a mercados, liberalización de inversión, compras gubernamentales, reglas de origen y derechos de propiedad intelectual, se incluyen temas como la competencia de las empresas estatales, la participación de las pequeñas y medianas empresas (pymes) en las cadenas globales de producción, los aspectos relacionados con el trabajo y el comercio, el desarrollo de los países y la coherencia regulatoria. Asimismo, el TPP pretende ser un acuerdo flexible que facilite la incorporación de nuevos miembros. Al final, el objetivo es avanzar hacia un acuerdo regional como el planteado en 2010 por los líderes del Foro Económico de Asia-Pacífico (APEC, por sus siglas en inglés) que involucraría a las 21 economías del mismo.

Por su parte, el RECEP es una iniciativa que se queda en Asia. Recién lanzada por la Asociación de Naciones del Sudeste Asiático (ANSEA) en 2012, este proyecto incluye, además de las 10 economías miembro de ANSEA, a Australia, China, Corea del Sur, India, Japón y Nueva Zelanda, países que ya tienen acuerdos bilaterales con esa asociación. Por el tamaño de su economía y por su relevancia en la región, China ejerce el liderazgo en este grupo. Este modelo de integración es menos demandante que el TPP y se está concentrando, por un lado, en los temas de acceso a mercados e inversión, dejando por fuera aquellos aspectos que pudieran ser más controversiales para estos países tan diversos en sus estrategias económicas; y por otro, pondrá énfasis en la cooperación y en la construcción de capacidades de las economías participantes.

La Alianza Pacífico es una iniciativa que, bajo el liderazgo de México, integra a tres países del Pacífico latinoamericano: Colombia, Perú y Chile, los cuales ya poseían entre sí tratados bilaterales. La AP busca, en primer lugar disminuir los costos de transacción al homologar las reglas del intercambio comercial entre sus miembros. Y segundo, la AP busca convertirse en la región líder en América Latina en fomentar los intercambios con Asia. De hecho, varios países de ambos lados del Pacífico se han interesado en participar como observadores en el avance de este proceso de negociación.

Sin duda, el dinamismo de la región de Asia-Pacífico se verá impulsado por estas tres modalidades de integración, sobre todo si se toma en cuenta que varios de los países participantes forman parte simultáneamente de las tres 
iniciativas. Es el caso de Australia, Malasia, Japón, Singapur, Nueva Zelanda y Vietnam, que participan al mismo tiempo en el TPP y en el RECEP. Mientras que México, Perú y Chile lo hacen en el TPP y en la AP.

No es de extrañar que, ante el estancamiento de las negociaciones de la Ronda de Doha de la Organización Mundial del Comercio (OMC), las tres iniciativas hayan rebasado a las negociaciones multilaterales, optando los países participantes por estrategias regionales más manejables y con mejores perspectivas de éxito. Es evidente también que, dada la multitud de acuerdos bilaterales existentes entre los países involucrados, éstos busquen facilitar el comercio entre sí al reducir los costos de transacción a los que se ven sometidas las empresas cuando, al querer hacer uso de las preferencias arancelarias, se topan con múltiples disposiciones dependiendo de las reglas de cada acuerdo bilateral. A esto hay que añadir las diferencias en las reglas de origen respecto a la procedencia de los insumos utilizados en los bienes finales que entran en los flujos de comercio. En fin, se trata de homologar reglas para superar el fenómeno del espagueti o noodle bowl.

Ahora bien, en cuanto a los beneficios de la integración, es de esperar que el ingreso y el empleo de los países participantes en cada uno de los tres procesos se incremente por el impacto del "efecto de la creación del comercio". Es decir, la mejor asignación de los recursos productivos regionales entre los socios con ventajas comparativas en determinados sectores o bienes, generará aumentos en la productividad y en el ingreso. Por supuesto, las ganancias obtenidas por cada país dependerán del mayor acceso a aquellos mercados que estaban más protegidos antes de entrar en vigor el acuerdo, así como también de los periodos de desgravación acordados. Por otra parte, la integración regional generará "desviación del comercio" al desplazar a países que no son miembros de uno de los tres convenios. Por ejemplo, el TPP tenderá a desplazar a China e India, que no gozarán de las preferencias otorgadas a los miembros de éste; lo mismo sucedería con Estados Unidos y el RECEP. Sin embargo, los países que participan en dos acuerdos a la vez, obtendrán mayores ganancias. Es el caso de Japón, que participa en el TPP y en el RECEP. En ese sentido, un reciente estudio de Petri, et al. (2012) hace un ejercicio econométrico muy detallado sobre las posibles ganancias para los países participantes en ambos procesos. ${ }^{4}$

4. Petri, Peter A., Plummer, Michael G., y Zhai, Fan (November 2012), The Trans-Pacific Partnership and Asia-Pacific Integration: A Quantitative Assessment, Wahington, DC: Peterson Institute for International Economics. 
Desde un punto de vista geopolítico, en un entorno global multipolar, Estados Unidos busca establecer un nuevo régimen de comercio en AsiaPacífico introduciendo nuevas reglas que le permitan consolidar su posición en la región. China, por su parte, hace lo propio con sus vecinos de Asia. Y los países de la Alianza Pacífico, que son las economías más abiertas de Latinoamérica, buscan asumir el liderazgo regional en las relaciones económicas con Asia. Un aspecto relevante de estos esfuerzos es que, entre más países se adhieran a las tres iniciativas, y entre más países participen en varias a la vez, se incrementan las posibilidades de que en el largo plazo resulte un gran acuerdo regional que genere menores desviaciones del comercio.

Aunque lo anterior sería el resultado "ideal", existe el riesgo de que no se llegue a lo planteado en cada una de las tres iniciativas. En el caso del TPP, que exige de sus miembros mayores concesiones, todavía después de 17 rondas de negociaciones (que no han incluido a Japón, pues fue el último país en incorporarse), los asuntos más "sensibles" se han ido dejando al último, pues los países esperan llegar a un intercambio "óptimo" en el que tengan que ceder lo menos. Estados Unidos tiene posturas ambivalentes en varios temas. Un ejemplo es el sector de lácteos que, ante las presiones de los grupos de interés internos, no quiere abrir su mercado a Nueva Zelanda con el argumento de que la empresa neozelandesa Fonterra, que es una cooperativa que controla el mercado en ese país, no juega bajo reglas de competencia. Al mismo tiempo, Estados Unidos exige que Canadá abra su mercado de lácteos, ya que en ese país existe un esquema de manejo de la oferta para los productos lácteos.

Por otra parte, en el caso del sector textil, las reglas de origen son un asunto de tensión, ya que para Vietnam ese sector es prioritario en su economía por lo que propone aplicar la regla de "corte y costura", para que el TPP no le afecte en los insumos que importa desde China. Mientras México y Perú, que han trabajado con Estados Unidos con la regla de "hilo en adelante", se oponen a la propuesta de Vietnam. En este último país el problema se complica, pues una sola empresa estatal controla $40 \%$ del mercado. Igualmente, los derechos de propiedad intelectual en productos farmacéuticos son otro tema tensión, ya que los países en desarrollo pugnan por menores periodos de protección de patentes, mientras que Estados Unidos desea prolongar tales periodos. Obviamente esto último incrementaría el precio de los medicamentos en los países menos desarrollados. Éstos son sólo unos cuantos ejemplos de un proceso muy complejo que al final puede llevar a un sinnúmero de excepciones que impediría llegar al óptimo de integración originalmente planteado. 
Con el RECEP, las metas son menos ambiciosas y ello permitiría llegar a consensos más fácilmente; sin embargo, esto no representaría un gran avance respecto a la situación prevaleciente en la actualidad con los acuerdos bilaterales. Más aún, las tensiones territoriales que se han presentado recientemente por la postura de China respecto a varias islas en disputa con sus vecinos, han puesto a estos últimos en alerta sobre las intenciones de su poderoso vecino. A ello hay que agregar las diferencias entre Japón, por un lado, y Corea del Sur y China, por otro, dado el nacionalismo que ha permeado al gobierno del primer ministro Abe. En esa línea, varios países de Asia ven con buenos ojos la mayor participación de Estados Unidos en la región, lo que puede dificultar el avance en los objetivos planteados.

Por su parte, la Alianza Pacífico es, de las tres modalidades de integración, la que avanza a paso más seguro, en especial por el menor número de participantes, lo acotado de sus metas, y el pragmatismo de la iniciativa.

En conclusión, lo cierto es que con el estancamiento del proceso multilateral en la OMC, las economías de Asia-Pacífico no se han quedado con los brazos cruzados y buscan esquemas, más o menos novedosos, para alcanzar estadios más profundos de integración que promuevan el comercio, la inversión y la cooperación entre sus miembros para alcanzar un mejor bienestar para la población. No obstante, que ello se logre dependerá al final de la presión que ejerzan los grupos de interés y de cómo los grupos negociadores logren acomodar esas preferencias frente a las de los demás países. Aun así, el primer paso está dado, una nueva ola de integración recorre el Océano Pacífico.

El presente número de la revista México y la Cuenca del Pacífico consta de cuatro artículos y una reseña. El primer trabajo expone que, tras la crisis financiera mundial iniciada en el año 2008, el funcionamiento de la economía internacional ha sido duramente cuestionado y que el origen de la crisis se ha convertido en objeto de debate político y académico. Como resultado, el déficit externo experimentado por la principal economía del orbe, Estados Unidos, ha sido señalado como uno de los detonantes de la misma. Así, el estudio sobre los determinantes del saldo negativo en la balanza de pagos de ese país ha adquirido relevancia en los últimos años. En esa línea, Ximena Valentina Echenique y Jorge Eduardo Mendoza, académicos de El Colegio de la Frontera Norte, analizan las causas de dicho déficit poniendo a prueba dos de las principales tesis que han sido utilizadas en la discusión académica, y comprueban, con base en un análisis econométrico, la existencia de una relación a largo plazo entre el desequilibrio externo de Estados Unidos y la 
adquisición de bonos y pagarés del Tesoro estadounidense por parte de China. Con lo anterior, concluyen que el desequilibrio macroeconómico actual está asociado con dos hechos generales, a saber: a) la conversión de las economías asiáticas en los principales acreedores internacionales, $\mathrm{y}$ b) las sobrevaluadas expectativas en relación con el crecimiento económico de Estados Unidos durante las últimas dos décadas. Asimismo, los autores rechazan el planeamiento que defiende una relación causal entre el ahorro y la inversión doméstica, y el déficit externo estadounidense.

José Jesús Bravo argumenta que el concepto de hegemonía, ampliamente discutido en la disciplina de las relaciones internacionales, generalmente ha sido entendido como el predominio de capacidades, en especial materiales, mediante las cuales un Estado construye y "gobierna" un orden internacional favorable a sus intereses. Sin embargo, en las últimas décadas los enfoques que destacan la dimensión social en la disciplina han ganado terreno y han contribuido notablemente al entendimiento de las dinámicas internacionales. En ese sentido, el autor parte de un enfoque constructivista para analizar el concepto, la construcción y el ejercicio de la hegemonía a partir del estudio de los casos de Gran Bretaña y Estados Unidos. Así, tras un breve recuento en torno al origen del concepto y a la interpretación que se le ha dado en distintas escuelas de las relaciones internacionales, el académico enfatiza que en ambos casos la posición hegemónica se alcanzó no sólo con base en las capacidades materiales, sino también a través de "la socialización de su poder". Es decir, por la manera en que han logrado el reconocimiento y la legitimidad de su posición por parte de los demás Estados. En ese tenor, Bravo subraya que, en conjunto con su poder naval, la promoción del libre comercio fue el elemento clave para la consecución de la Pax Britannica; entre tanto, para Estados Unidos el impulso de normas internacionales de interés común, mediante la práctica del multilateralismo, fue fundamental para generar estabilidad internacional y para consolidar su poder. Más aún, el autor destaca que el abandono de dicha práctica, cuando ha ocurrido, ha minado la hegemonía estadounidense y que, por tanto, el avance hacia un contexto cada vez más incluyente es vital para fortalecer su posición hegemónica.

Daniel Efrén Morales examina la importancia económica y política del Foro BRICS (Brasil, Rusia, India, China y Sudáfrica) frente a la reciente crisis económica mundial, y contrasta sus políticas económicas con las del G7 (Estados Unidos, Japón, Alemania, Francia, Reino Unido, Canadá e Italia) en el contexto de lo que el mismo autor denomina como la "gran recesión". 
En la primera parte de su trabajo Morales analiza a las potencias mundiales del G7 en su calidad de Estados centrales y las políticas económicas que han promovido a través de los principales organismos financieros internacionales; posteriormente examina a las potencias regionales del BRICS a partir de su naturaleza semiperiférica, su creciente protagonismo en la economía mundial y sus posturas frente al orden internacional actualmente en crisis; en la última parte de su trabajo explica y compara las acciones emprendidas por las naciones del G7 y el Foro BRICS para gestionar la crisis económica internacional actual.

Cao Yufei, por su parte, explica en su trabajo la pronunciación correcta del idioma chino desde el punto de vista de la función social del lenguaje: la comunicación. Basándose en el análisis contrastivo de los rasgos prosódicos distintivos entre el idioma chino y el español, la autora propone algunos consejos prácticos para mejorar la enseñanza de la fonética del chino a hispanohablantes. En el marco de la fonética acústica, para Yufei los rasgos distintivos se refieren a las relaciones entre las unidades fonéticas distintivas de las lenguas, que se basan en la presencia o ausencia de uno o varios rasgos distintivos, mismos que se dividen en rasgos prosódicos y rasgos intrínsecos o inherentes. La autora distingue tres tipos de rasgos prosódicos: la frecuencia fundamental, la intensidad y la cantidad (también denominados tono, fuerza y cantidad, respectivamente). Desde el punto de vista de la fonética auditiva, Yufei sugiere que, en la percepción de un estímulo acústico, existen dos aspectos de naturaleza muy diferentes: uno es el físico, que se puede medir objetivamente en todos sus componentes; otro es el psicológico, esto es, el grado de sensación que ese estímulo produce en nosotros; y que este aspecto sensorial es subjetivo y mucho más difícil de controlar.

Expresamos nuestro sincero agradecimiento a todos nuestros colaboradores por el apoyo brindado a México y la Cuenca del Pacífico, a los miembros tanto del Consejo Editorial como del Comité Editorial por su generosa e invaluable labor para preservar la calidad de la revista, y al Centro Universitario de Ciencias Sociales y Humanidades de la Universidad de Guadalajara por el apoyo para la publicación de la misma. 


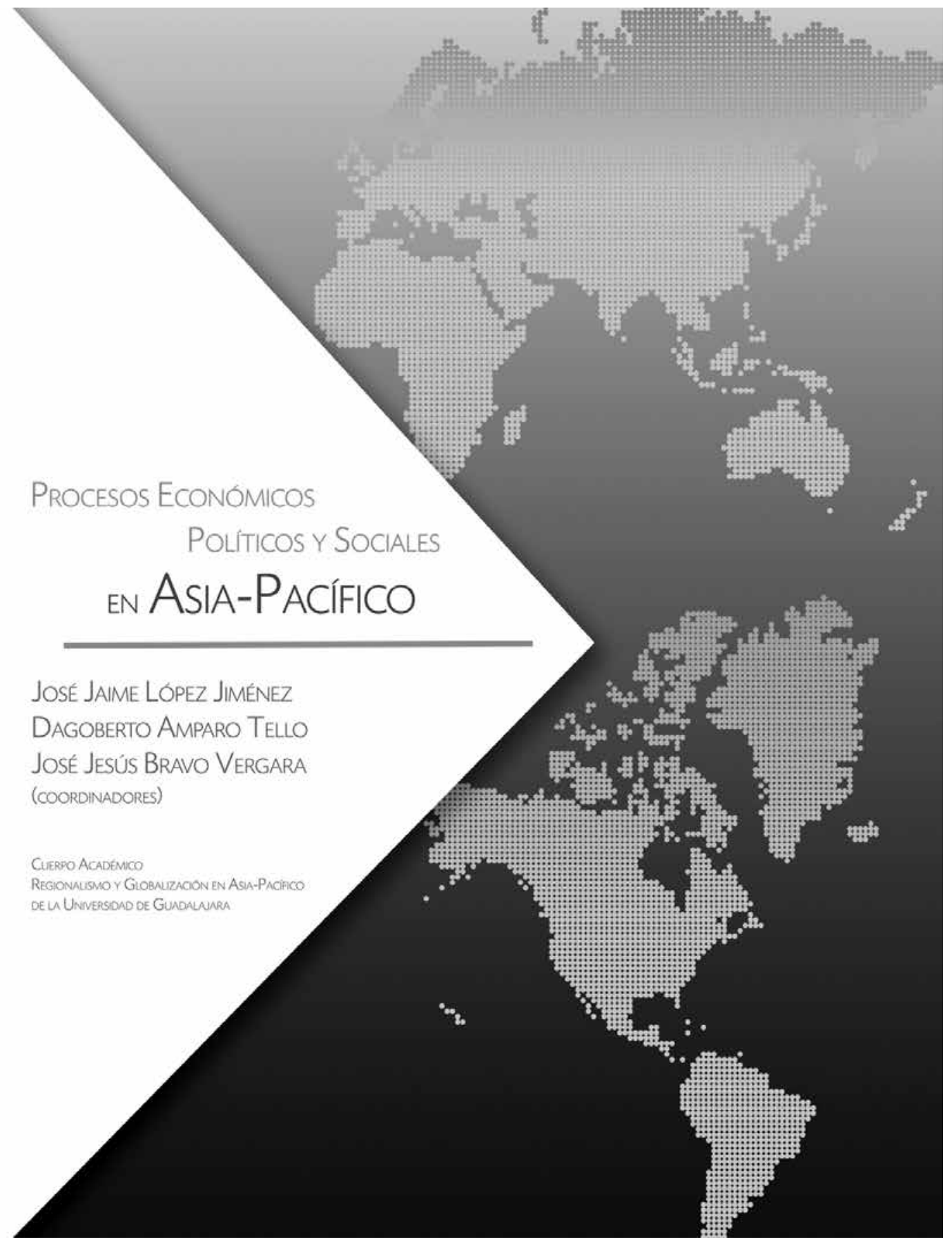

